\title{
Adjuvant Fuzheng Huayu Capsule Reduces the Incidence of Hepatocellular Carcinoma in Patients with Hepatitis B-Caused Cirrhosis
}

\author{
Ke Shi, ${ }^{1,2}$ Yao Liu, ${ }^{1}$ Xiaojing Wang, ${ }^{1}$ Yuxin Li, ${ }^{1}$ Qun Zhang, ${ }^{1}$ Ying Hu, ${ }^{1}$ Chongping Ran, \\ Yunyi Huang, ${ }^{1,2}$ Jie Hou, ${ }^{1,2}$ and Xianbo Wang $\mathbb{D}^{1}$ \\ ${ }^{1}$ Center of Integrative Medicine, Beijing Ditan Hospital, Capital Medical University, Beijing, China \\ ${ }^{2}$ Department of Gastroenterology, Dongzhimen Hospital, Beijing University of Chinese Medicine, Beijing, China \\ Correspondence should be addressed to Xianbo Wang; wangxb@ccmu.edu.cn
}

Received 11 August 2020; Revised 28 September 2020; Accepted 16 October 2020; Published 29 October 2020

Academic Editor: JiaTuo Xu

Copyright (C) $2020 \mathrm{Ke}$ Shi et al. This is an open access article distributed under the Creative Commons Attribution License, which permits unrestricted use, distribution, and reproduction in any medium, provided the original work is properly cited.

\begin{abstract}
Aim. Fuzhenghuayu (FZHY) capsule can inhibit the progression of cirrhosis. This study explored whether FZHY can reduce the incidence of hepatocellular carcinoma (HCC) in patients with hepatitis B-caused cirrhosis (HBC) undergoing antiviral therapy. Methods. A retrospective review of 842 patients with HBC between 2011 and 2015 was performed, including 270 treated with FZHY combined with nucleos ( $\mathrm{t}$ ) ide analogues (NAs) and 572 with NAs alone. The incidence of HCC was compared between the FZHY ( $n=259)$ and control $(n=259)$ groups using $1: 1$ propensity score (PS) matching. The incidence of HCC in patients with HBC with different Child-Turcotte-Pugh (CTP) classifications and Toronto HCC risk index (THRI) scores was analyzed using Kaplan-Meier curves. Results. The 5-year cumulative incidence of HCC before and after PS matching was 151 (17.9\%) and 86 (16.6\%), respectively. In PS-matched samples, the multivariate Cox proportional-hazards model indicated that the FZHY group demonstrated a significantly lower risk for HCC than the control group (adjusted hazard ratio [aHR] $=0.32$, 95\% CI 0.19-0.53 $P<0.001)$. The risk of HCC diminished with increased duration of FZHY use. The stratified analysis revealed that the FZHY group, regardless of CTP classification, benefited significantly from FZHY therapy. Patients in the medium- and high-THRI risk groups were the dominant population for FZHY. Conclusions. FZHY combined with NAs was associated with a significantly lower risk of HCC than NAs alone in patients with HBC, which supports the integration of FZHY with antiviral treatment into clinical practice.
\end{abstract}

\section{Introduction}

Cirrhosis is the most significant risk factor for the development of hepatocellular carcinoma (HCC). The 5-year cumulative incidence of HCC in patients with hepatitis B virus-caused cirrhosis (HBC) has been reported to be $17 \%$ in East Asia and 10\% in Western countries [1]. The risk of HCC development in patients infected with hepatitis $\mathrm{B}$ virus is $10-$ to 100 -fold greater than in uninfected individuals [2]. In China, more than $80 \%$ of HCC cases are associated with hepatitis $\mathrm{B}$ virus infection. The majority of these patients (80-95\%) have cirrhosis at the time of diagnosis of HCC [3]. HCC has become the third most common cause of cancer- related death with a 5-year survival rate of approximately $35 \%[4,5]$. Hence, effective preventive measures and therapies are necessary to slow the progression of cirrhosis to HCC.

Currently, the recommended first-line options for the treatment of HBC are entecavir (ETV) or tenofovir (TDF) [6]. Studies comparing ETV or TDF with older NAs did not observe a difference in HCC risk reduction between the agents $[7,8]$. Studies have found that patients treated with ETV remain at considerable risk for developing HCC despite long-term viral suppression [9]. Some studies reported that the annual incidence of HCC ranges from $0.9 \%$ to $5.4 \%$ in patients with $\mathrm{HBC}$ treated with ETV or TDF [10]. These data 
indicate that the risk for HCC can be reduced but not eliminated, probably due to risk factors that cannot be modified by antiviral therapy.

In China, traditional Chinese medicine (TCM) has been used to treat chronic liver diseases for centuries [11] and is now a component of alternative therapies and is still used extensively. The severity of liver fibrosis is another risk factor for HCC [12]. Fuzheng Huayu (FZHY) capsule, an effective antifibrosis TCM, has been investigated in both animal experiments $[13,14]$ and clinical trials [15] for several years. FZHY has demonstrated benefits in patients with cirrhosis, who have experienced significant improvement in liver function and quality of life through decreased collagen synthesis, increased degradation of the extracellular matrix, and reversal of hepatic fibrosis [16]. Song et al. showed that compared with placebo, FZHY could improve clinical symptoms in patients with $\mathrm{HBC}$ [15]. A previous study also indicated that the use of TCM is related to decreased HCC risk in patients with chronic hepatitis B [17]. However, few longterm population-based studies have compared the combined application of FZHY and NAs with NAs alone on HCC development among patients with varying clinical stages of $\mathrm{HBC}[15,17]$. Additionally, it remains unclear whether FZHY can prevent and/or reduce the incidence of HCC.

Accordingly, we aimed to investigate whether FZHY combined with antiviral therapy can reduce the incidence of HCC in patients with HBC and to analyze the role of FZHY in different populations to provide important clinical evidence supporting adjuvant therapy.

\section{Materials and Methods}

2.1. Research Subjects. We retrospectively enrolled 1,595 patients diagnosed with $\mathrm{HBC}$ at the Beijing Ditan Hospital of Capital Medical University (Beijing, China) between January 2011 and January 2015. Patients diagnosed with HBC for the first time, aged $>18$ and $<80$ years, and undergoing antiviral treatment with ETV or TDF were included. Individuals infected with human immunodeficiency virus, those with other hepatitis infections (including A, C, D, and E), those with HCC diagnosed before the baseline date, those who underwent liver failure or liver transplantation, those with active alcoholism or severe fatty liver, and those who died within 5-year or lost follow-up were excluded. A total of 842 patients with compensated and decompensated cirrhosis were eventually included. Of them, 270 received FZHY for more than 6 months and 572 did not receive FZHY. Patients were divided into two cohorts according to whether they received combined FZHY (FZHY group, $n=259$ ) or no combined FZHY (control group, $n=259$ ) by 1:1 PSmatched analysis (Figure 1). FZHY group was defined as the administration of at least one course of FZHY $(\geq 6$ months) based on antiviral therapy. This study protocol conformed to the ethical guidelines of the Declaration of Helsinki and was approved by the ethics committee of the hospital.
2.2. Clinical Definitions. The baseline date was defined as the date of the first diagnosis of cirrhosis in the hospital. The endpoint of this study was the new diagnosis of HCC or the end of the 5-year follow-up. Compensatory cirrhosis was diagnosed as follows: (1) pathological (F4 on biopsy); (2) esophageal varices on endoscopy, exclusion of noncirrhotic portal hypertension; (3) In the absence of histological, endoscopic, two of three criterions should be met:(1) ultrasonography, computed tomography, or magnetic resonance imaging result indicated imaging changes in liver morphology, such as nodules in the hepatic parenchyma and serrated change on the liver surface; (2) platelet count $<100 \times 10^{9}$ cells/L, without other causes; (3) serum albumin $<35.0 \mathrm{~g} / \mathrm{L}$, international normalized ratio $>1.3$, or prothrombin time prolonged $>3$ seconds. The diagnosis of decompensated cirrhosis is based on cirrhosis with portal and venous hypertension complications and/or liver dysfunction. (1) Having the diagnosis basis of cirrhosis; (2) having the complications related to portal hypertension, such as ascites, esophageal, and gastric varices bleeding, hepatic encephalopathy [18]. HCC was diagnosed by standard histological and/or compatible radiological findings. Diagnosis is based on imaging techniques obtained by multiple detector CT scan or dynamic contrast-enhanced MRI. The diagnosis can be established if the typical vascular hallmarks of HCC (hypervascularity in the arterial phase with washout in the portal venous or delayed phase) are identified in a nodule of $>1 \mathrm{~cm}$ diameter using one of these two modalities [2]. Virological response (VR) was defined as an undetectable HBV DNA load at the time of HCC development or at the end of follow-up. Routine laboratory investigations, including alpha-fetoprotein and radiological examination, were performed every 3-6 months.

2.3. Study Medications. FZHY refers to the herbal extraction, which comprises Danshen (Radix salvia miltiorrhizae), Chongcao (Cordyceps), Taoren (Semen persicae), Jiaogulan (Gynostemma pentaphyllum), Songhuafen (Pollen pini), and Wuweizi (Fructus schisandrae chinensis) (Table 1). All ingredients of FZHY have been approved by the State Food and Drug Administration (SFDA) of China. FZHY capsule is suitable for patients with chronic hepatitis $B$ hepatic fibrosis.

From the above six herbs, Dan shen, Taoren, and Jiaogulan were taken, and they were decocted twice and water was added 10 times and 8 times, respectively (the first time for 2 hours, and the second time for 1.5 hours). Then, the filtrate was concentrated to a relative density of about $1.20\left(50^{\circ} \mathrm{C}-55^{\circ} \mathrm{C}\right)$ and cooled, and then ethanol was added under mixing to make the alcohol content to $70 \%$. They were then allowed to settle, and the supernatant was taken. After filtration, the filtrate was concentrated to a relative density of 1.1-1.2 $\left(50^{\circ} \mathrm{C}-55^{\circ} \mathrm{C}\right)$ and allowed to settle. Further, Chongcao and Wuweizi were taken, and the mixture was heated and refluxed twice and $70 \%$ ethanol was added 10 times and 8 times, respectively (the first time for 1.5 hours, and the second time for 1 hour), and the reflux was then combined and filtered. The filtrate was concentrated to a relative density of $1.1-1.2\left(50^{\circ} \mathrm{C}-55^{\circ} \mathrm{C}\right)$ and kept. Then, 


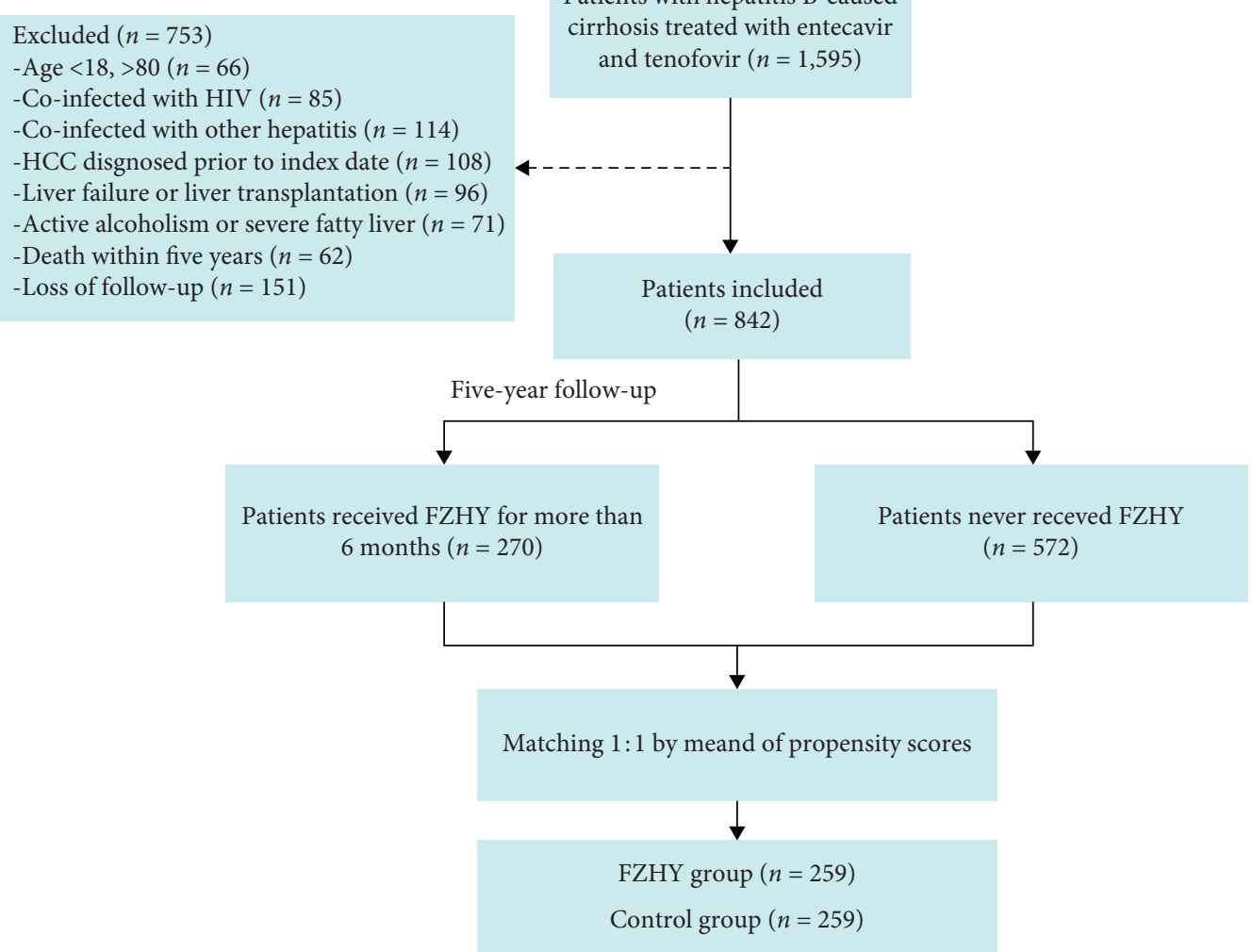

FIGURE 1: Flowchart of the enrollment of patients with hepatitis B-caused cirrhosis.

TABLE 1: The ingredients contained in Fuzheng Huayu (FZHY) capsule.

\begin{tabular}{lccc}
\hline TCM name & Latin name & Chinese name & Picture \\
\hline & Radix salvia miltiorrhizae & Danshen & Weight $(\mathrm{g})$ \\
\hline FZHY & Cordyceps & Chongcao & Taoren \\
& Gynostemma pentaphyllum & Jiaogulan & Songhuafen \\
& Pollen pini & Wuweizi & 166 \\
& Fructus schisandrae chinensis &
\end{tabular}

Songhuafen was soaked in $50^{\circ} \mathrm{C} 2$ times and $50 \%$ ethanol was added 10 times and 8 times, respectively (the first time for 4 hours, and the second time for 2 hours), and then it was combined to the extract and concentrated to a relative density of $1.1-1.2\left(50^{\circ} \mathrm{C}-55^{\circ} \mathrm{C}\right)$. It was then combined with the above two spare concentrates and dried, and a proper amount of auxiliary materials was then added, mixed, crushed into powder, and placed into a capsule $(0.3 \mathrm{~g} /$ capsule). Z20020073 represents the approval number of national medicine permission number (NMPN) of FZHY capsule (Huanghai Pharmaceutical Co., Ltd., Shanghai, China). The total daily dose of FZHY ( $0.3 \mathrm{~g}$ per capsule) was 15 capsules, administered orally 3 times per day after meals.
2.4. Statistical Analysis. Statistical analysis was performed using SPSS version 25 (IBM Corporation, Armonk, NY, USA). A two-sided $P<0.05$ was considered statistically significant. Normally distributed continuous variables were compared using the $t$-test while the Mann-Whitney $U$ test was used for nonnormally distributed variables. Categorical variables were analyzed using the chi-squared test or Fisher's exact test, as appropriate. The crude and adjusted hazard ratio ( $\mathrm{HR}$ and aHR, respectively) and corresponding 95\% confidence interval (CI) were computed and interpreted accordingly. The cumulative incidence of HCC was computed using the Kaplan-Meier curve analysis, and the logrank test was used to test the difference in the incidence of 
HCC between groups. Analysis stratified according to FZHY use was performed using the Cox proportional-hazards regression model to assess the relative risk for HCC between the FZHY group and the control group.

To overcome the effects of possible confounders, propensity scores (PS) were calculated using logistic regression based on age, sex, alcohol consumption, diabetes, hypertension, hepatitis $\mathrm{B} e$ antigen, albumin, total bilirubin, gamma-glutamyl transferase, white blood cell count, platelet count, creatinine, prothrombin activity, international normalized ratio, alpha-fetoprotein, HBV DNA, Child-Turcotte-Pugh (CTP) class, and model for end-stage liver disease. The FZHY group was matched with the control group according to the generated propensity scores using a one-to-one nearest-neighbor caliper of width 0.02 .

\section{Results}

3.1. Baseline Characteristics. Among 842 patients with HBC treated with either ETV or TDF, 151 developed HCC within 5 years. Before PS matching, 270 (32.1\%) received FZHY for $\geq 6$ months during the study period and 572 (67.9\%) did not receive FZHY. FZHY group exhibited a higher rate of diabetes, hypertension, total bilirubin, gamma-glutamyl transferase, white blood cell count, prothrombin activity, and alpha-fetoprotein than the control group. In addition, the FZHY group exhibited lower creatine levels (Table 2).

Among the 259-pair PS-matched population, baseline characteristics were balanced between patients in the two groups. The median age of FZHY and control group was 50 (interquartile range [IQR] 42-59) and 49 (IQR 42-58) years, respectively. A total of $86 \mathrm{HCC}$ events occurred among all study participants, including in 60 control groups and 26 FZHY groups. The cumulative 1-, 3- and 5-year rates of HCC were $0.4 \%, 6.9 \%$, and $10.0 \%$ in FZHY group and $5.0 \%$, $18.1 \%$, and $23.2 \%$ in control group, respectively. VR was achieved in $93 \%$ of the FZHY group and $96 \%$ of the control group at 1 year after ETV/TDF therapy and in $98.8 \%$ and $99 \%$ of those at the end of the follow-up, respectively.

3.2. Effect of FZHY on HCC Occurrence. Before and after PS matching, the 5-year cumulative incidence of HCC was 151 $(17.9 \%)$ and 86 (16.6\%), respectively. Kaplan-Meier curve analysis revealed that the cumulative incidence of HCC during the 5-year follow-up period was significantly lower in the FZHY group than in the control group, regardless of whether PS matching was performed (all $P<0.001$ ) (Figures 2(a) and 2(b)).

FZHY group was divided into three subgroups based on the time of using FZHY: $<12,12-36$, and $>36$ months. The risk of HCC according to the three subgroups was negatively correlated with time of cumulative use. Both before and after PS matching, Kaplan-Meier curve analysis revealed a lower incidence of HCC in the FZHY group who took FZHY for $>12$ months than in the control group (all $P<0.001$ ) (Figures 2(c) and 2(d)). The multivariate Cox proportionalhazards model revealed that after PS matching, the adjusted risk of HCC in FZHY group was significantly lower than that in the control group (aHR 0.32, 95\% CI 0.19-0.53; $P<0.001)$. FZHY group with $12-36$ months of FZHY treatment were at a significantly lower risk of HCC (aHR $0.28,95 \%$ CI $0.15-0.54 ; P<0.001$ ), as were FZHY users with $>36$ months of FZHY (aHR 0.04, 95\% CI 0.01-0.70; $P=0.028$ ) (Table 3 ). Thus, the longer the duration of active FZHY therapy, the lower the incidence of HCC.

\subsection{Subgroup Analysis of the Risk for the Development of HCC.} The Toronto HCC risk index (THRI), which is based on age, sex, etiology of liver disease, and platelet count, was developed to predict the risk of HCC in patients with cirrhosis. The score performs well and has been externally validated (Supplementary Table 1) [19]. In this study, most patients (98.6\%) were in the medium- (120-240) and high-risk (>240) groups of the THRI score. Only 7 patients were in the low-risk group (FZHY group $[n=2]$, control group $[n=5]$ ), none of whom had HCC in 5 years. Regardless of the risk group (i.e., medium or high), the FZHY group demonstrated a significantly lower risk than the control group (mediumrisk group, 1.6\% versus [vs.] 19.4\%; high-risk group, $12.8 \%$ vs. $25.1 \%$; all $P=0.001$ ) (Figures 3(b) and 3(c)) and also had a lower risk of HCC regardless of CTP classification (all $P<0.05$ ) (Figures 3(d)-3(f)).

\section{Discussion}

Cirrhosis is a premalignant state with an increased risk for HCC over time [20]. The fibrotic burden is becoming one of the most important factors related to HCC in patients with $\mathrm{HBC}$, especially in those with sustained low viral status [21]. Antifibrosis therapy is an important way to effectively prevent progression to cirrhosis or HCC [11]. There are currently no effective Food and Drug Administration-approved antifibrosis drugs. Thus, many patients use complementary and alternative therapies to protect hepatic function, prevent disease progression, and inhibit the development of HCC.

Accumulating evidence has indicated that FZHY combined with antiviral therapy yields better effects in treating HBV-related cirrhosis, with no serious adverse events [22]. In the present retrospective, population-based cohort study, our results demonstrated that the administration of FZHY was a protective factor for 5-year HCC development in patients with $\mathrm{HBC}$ treated with ETV or TDF. The cumulative incidence of HCC in the FZHY group was lower than that in the control group after PS matching $(P<0.001)$. We also found significant relationships between treatment courses and efficiency between the use of FZHY and HCC in patients with HBC. The adjusted HR for FZHY use was 0.66 for $<12$ months, 0.28 for $12-36$ months, and 0.04 for $>36$ months, indicating that using of FZHY over longer periods would lead to a stronger protective effect.

As a parameter of compensatory liver function, the CTP score has been used to evaluate the efficacy of TCM [23]. Some studies have demonstrated the good therapeutic efficacy of FZHY, which can also improve CTP score in those with HBC [15]. In the present study, FZHY users benefited significantly 
TABLE 2: Clinical characteristics of patients with hepatitis B-caused cirrhosis before and after propensity score matching.

\begin{tabular}{|c|c|c|c|c|c|c|}
\hline \multirow[b]{2}{*}{ Variable } & \multicolumn{3}{|c|}{ Before propensity matching } & \multicolumn{3}{|c|}{ After propensity matching } \\
\hline & $\begin{array}{l}\text { TCM user } \\
(n=270)\end{array}$ & $\begin{array}{l}\text { Non-TCM users } \\
(n=572)\end{array}$ & $\begin{array}{c}P \\
\text { value }\end{array}$ & $\begin{array}{l}\text { TCM user } \\
(n=259)\end{array}$ & $\begin{array}{c}\text { Non-TCM users } \\
(n=259)\end{array}$ & $\begin{array}{c}P \\
\text { value }\end{array}$ \\
\hline Age, years & $50.0(42.0,59.0)$ & $50.0(42.0,58.0)$ & 0.525 & $50.0(42.0,59.0)$ & $49.0(42.0,58.0)$ & 0.234 \\
\hline Male sex, $n(\%)$ & $191(70.7 \%)$ & $393(68.7 \%)$ & 0.550 & $184(71.0 \%)$ & $168(64.8 \%)$ & 0.132 \\
\hline Family history of HCC, $n(\%)$ & $19(7.0 \%)$ & $33(5.8 \%)$ & 0.476 & $18(6.9 \%)$ & $16(6.2 \%)$ & 0.723 \\
\hline Smoking, $n(\%)$ & $61(22.6 \%)$ & $128(22.4 \%)$ & 0.944 & $58(22.4 \%)$ & $58(22.4 \%)$ & 1.000 \\
\hline Alcohol consumption, $n(\%)$ & $41(15.2 \%)$ & $85(14.9 \%)$ & 0.984 & $39(15.1 \%)$ & $39(15.1 \%)$ & 1.000 \\
\hline Diabetes, $n(\%)$ & $57(21.1 \%)$ & $85(14.9 \%)$ & 0.024 & $53(20.5 \%)$ & $49(18.9 \%)$ & 0.659 \\
\hline Hypertension, $n(\%)$ & $55(20.4 \%)$ & $80(13.9 \%)$ & 0.018 & $50(19.3 \%)$ & $44(16.9 \%)$ & 0.494 \\
\hline Ascites, $n(\%)$ & $46(17.0 \%)$ & $77(13.4 \%)$ & 0.170 & $45(17.3 \%)$ & $41(15.8 \%)$ & 0.637 \\
\hline Hepatic encephalopathy, $n(\%)$ & $14(5.2 \%)$ & $18(3.1 \%)$ & 0.149 & $14(5.4 \%)$ & $10(3.9 \%)$ & 0.403 \\
\hline Gastroesophageal varices, $n(\%)$ & $69(25.5 \%)$ & $137(23.9 \%)$ & 0.613 & $65(25.1 \%)$ & $81(31.3 \%)$ & 0.118 \\
\hline HBeAg positivity, $n(\%)$ & $143(52.9 \%)$ & $296(51.7 \%)$ & 0.818 & $139(53.6 \%)$ & $126(48$. & 0.206 \\
\hline CTP score, $n(\%)$ & $8.0(6.0,10.0)$ & $8.0(6.0,10.0)$ & 0.837 & $8.0(6.0,10.0)$ & $8.0(6.0,10.0)$ & 0.130 \\
\hline MELD score, $n(\%)$ & $10.3(8.2,13.2)$ & $10.3(8.3,12.9)$ & 0.822 & $10.3(8.3,13.2)$ & $10.7(8.4,13.6)$ & 0.573 \\
\hline Alanine aminotransferase, U/L & $37.9(24.1,80.2)$ & $36.4(24.0,76.5)$ & 0.744 & $37.7(24.1,78.3)$ & $37.0(27.2,114.5)$ & 0.139 \\
\hline $\begin{array}{l}\text { Aspartate aminotransferase, U/ } \\
\text { L }\end{array}$ & $43.3(30.8,76.7)$ & $42.9(29.1,85.3)$ & 0.622 & $43.3(31.5,76.5)$ & $46.5(31.9,111.0)$ & .132 \\
\hline Total bilirubin, $\mu \mathrm{mol} / \mathrm{L}$ & $27.1(18.6,44.8)$ & $23.7(14.6,39.7)$ & 0.004 & $27.2(18.9,44.6)$ & $29.5(16.7,48.0)$ & 0.838 \\
\hline Albumin, $g / L$ & $32.2(27.9,37.1)$ & $32.2(27.7,37.5)$ & 0.798 & $32.4(27.9,37.0)$ & $32.2(27.4,36.4)$ & 0.329 \\
\hline $\begin{array}{l}\text { Gamma-glutamyl transferase, } \\
\text { U/L }\end{array}$ & $50.3(24.7,90.9)$ & $35.7(20.3,83.9)$ & 0.010 & $50.0(24.9,90.8)$ & $38.5(20.8,85.1)$ & 0.102 \\
\hline White blood cell count, $\times 10^{9} / \mathrm{L}$ & $4.2(3.2,5.4)$ & $3.6(2.6,5.1)$ & 0.001 & $4.0(3.1,5.3)$ & $3.8(2.7,5.7)$ & 0.278 \\
\hline Platelets, $\times 10^{9} / \mathrm{L}$ & $71.0(50.4,101.0)$ & $68.0(47.2,99.0)$ & 0.715 & $71.0(50.0,101.0)$ & $69.8(51.8,101.0)$ & 0.731 \\
\hline Creatinine, $\mu \mathrm{mol} / \mathrm{L}$ & $64.0(53.0,73.4)$ & $65.9(56.3,75.0)$ & 0.027 & $64.4(53.5,73.9)$ & $63.0(55.0,72.0)$ & 0.737 \\
\hline Blood urea nitrogen, $\mathrm{mmol} / \mathrm{L}$ & $5.2(4.2,6.8)$ & $5.8(4.1,6.9)$ & 0.644 & $5.2(4.2,6.9)$ & $5.2(3.8,6.8)$ & 0.367 \\
\hline International normalized ratio & $1.2(1.1,1.3)$ & $1.2(1.1,1.4)$ & 0.673 & $1.2(1.1,1.3)$ & $1.2(1.1,1.3)$ & 0.726 \\
\hline Prothrombin activity,\% & $61.0(50.0,76.0)$ & $60.0(48.0,68.0)$ & 0.021 & $61.0(50.0,76.0)$ & $61.0(50.0,73.0)$ & 0.811 \\
\hline Alpha-fetoprotein, $\mathrm{ng} / \mathrm{ml}$ & $10.0(4.0,64.4)$ & $6.4(3.2,33.0)$ & 0.006 & $10.0(4.0,63.5)$ & $9.0(4.2,47.9)$ & 0.926 \\
\hline HBV DNA, $\log _{10} \mathrm{IU} / \mathrm{ml}$ & $4.2(2.7,5.7)$ & $3.8(2.7,5.6)$ & 0.118 & $4.1(2.7,5.7)$ & $4.0(2.7,5.7)$ & 0.881 \\
\hline
\end{tabular}

Data are presented as $n$ (\%) or median (interquartile range). CTP: Child-Turcotte-Pugh; MELD: Model for End-Stage Liver Disease.

from antifibrosis therapy, regardless of CTP classification. The THRI has demonstrated good predictive ability for HCC in patients with cirrhosis and has been validated in an external cohort [19]. FZHY group had a lower risk of HCC than the control group, especially in patients with $\mathrm{HBC}$ in the mediumor high-risk groups of THRI. Overall, the results of this study add to the evidence supporting the potential role of FZHY in preventing-or, at least, slowing - the development of HCC. These findings may be beneficial to clinicians in providing more effective treatment strategies to improve clinical outcomes.

HCC is strongly associated with liver fibrosis and cirrhosis, with approximately $80-90 \%$ of patients with HCC exhibiting underlying fibrosis [24]. Chronic liver injury causes hepatocyte cell death, fibrosis, and, ultimately, the development of HCC [25]. Several studies have shown that a high fibrosis index and liver stiffness, which are indirect measurements of liver fibrosis, are positively correlated with the risk of HCC $[26,27]$. Based on proven mechanisms, we analyzed the antifibrotic effect of FZHY in reducing the incidence of HCC, which was as follows: (1) Regulation of the signal transduction pathway: FZHY directly regulates many important pathways, such as the p53 signaling pathway, which correlates with various types of cancer [28]. (2) Regulation of cytokine expression: Transforming growth factor $\beta 1$ (TGF- $\beta 1$ ) expression level correlated with all stages of disease progression, from fibrosis to cirrhosis and HCC.
FZHY remarkably reduces TGF- $\beta 1$ levels [13]. (3) Inhibition of inflammatory response: Chronic inflammation initiates fibrosis and cancer. FZHY inhibits the expression of tumor necrosis factor $\alpha(\mathrm{TNF} \alpha)$ and interleukin 6 (IL-6) [14]. (4) Upregulation of the tumor suppressor gene and downregulation of oncogene expression: The previous study has shown that FZHY reduces the expression of Bax, increases the expression of $\mathrm{Bcl}-2$, and decreases the apoptosis of hepatocytes [29]. (5) Inhibition of angiogenesis: Vascular endothelial growth factor (VEGF) is an important factor in tumor growth. It is reported that FZHY can reduce the expression of HIF-1 $\alpha$, VEGF, and its receptor [16]. (6) The anti-HCC effects of FZHY may also be due to its regulation of the immune system [30]. Together, these actions may reduce the risk of fibrosis-related carcinogenesis.

To the best of our knowledge, this is the first observational study to investigate the effectiveness of FZHY in patients with multiple clinical stages of $\mathrm{HBC}$ by providing real-world evidence. There were, however, a few limitations to our study, the first of which was its retrospective, single-center cohort design, which may have introduced bias in calculating differences in clinical characteristics between the FZHY and the control groups. To address these confounders, patients were matched according to the PS score to eliminate confounding factors. Further prospective randomized controlled trials aiming to assess 


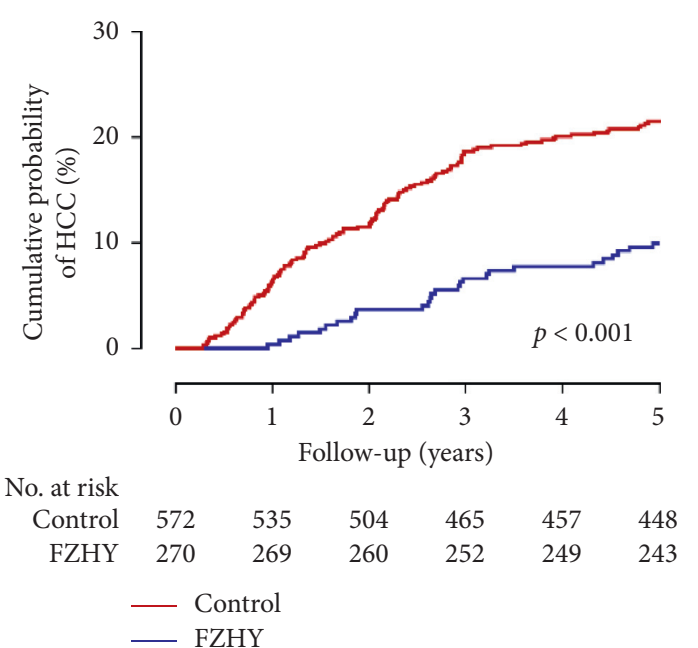

(a)

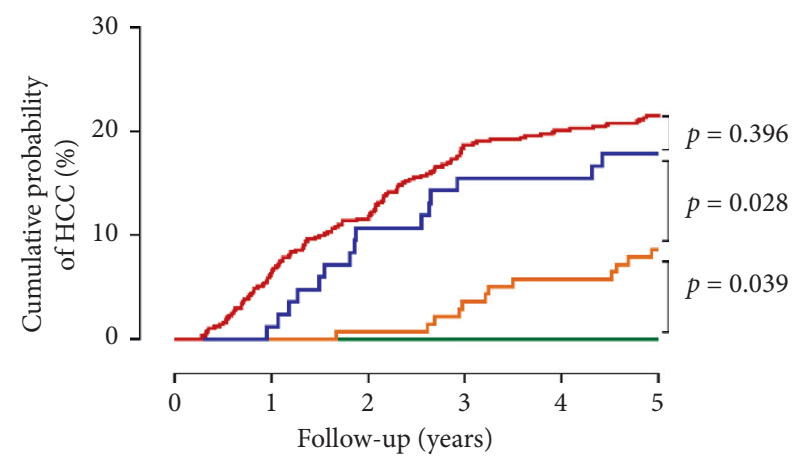

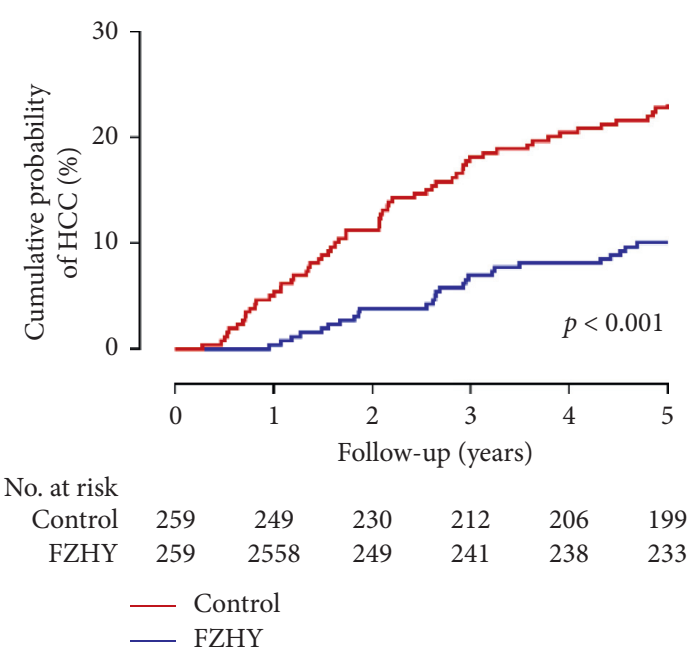

(b)
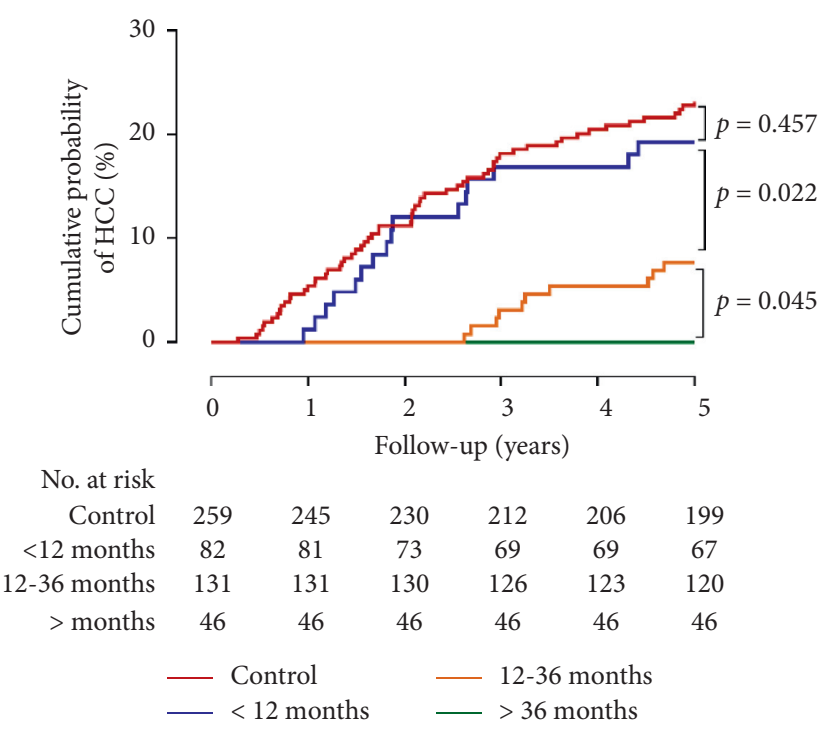

(d)

FIGURE 2: Cumulative incidence of HCC in patients with hepatitis B-caused cirrhosis before (a) and after matching with those with and without FZHY use. (b) Cumulative incidence of HCC in patients before (c) and after matching (d) for different times of FZHY use. HCC: hepatocellular carcinoma; FZHY: Fuzheng Huayu capsule.

TABLE 3: Risk of hepatocellular carcinoma according to the cumulative use of FZHY among patients with hepatitis B-caused cirrhosis.

\begin{tabular}{|c|c|c|c|c|c|c|}
\hline \multirow{2}{*}{ No. of FZHY days } & \multirow{2}{*}{$\begin{array}{c}\text { Total } \\
(n=518)\end{array}$} & \multirow{2}{*}{$\begin{array}{c}\text { HCC } \\
(n=86)\end{array}$} & \multicolumn{4}{|c|}{ Hazard ratio $(95 \% \mathrm{CI})$} \\
\hline & & & \multicolumn{2}{|c|}{ Crude $^{\dagger}$} & \multicolumn{2}{|c|}{ Adjust* } \\
\hline Control group & 259 & 60 & \multicolumn{2}{|c|}{ Reference } & \multicolumn{2}{|c|}{ Reference } \\
\hline FZHY group & 259 & 26 & $0.39(0.25-0.62)$ & $P<0.001$ & $0.32(0.19-0.53)$ & $P<0.001$ \\
\hline$<12$ months & 82 & 15 & $0.77(0.44-1.37)$ & $P=0.381$ & $0.66(0.35-1.24)$ & $P=0.197$ \\
\hline 12-36 months & 131 & 11 & $0.32(0.18-0.62)$ & $P=0.001$ & $0.28(0.15-0.54)$ & $P<0.001$ \\
\hline$>36$ months & 46 & 0 & $0.04(0.01-0.70)$ & $P=0.028$ & $0.04(0.01-0.70)$ & $P=0.028$ \\
\hline
\end{tabular}

Data are presented as hazard ratios (95\% confidence intervals). ${ }^{*}$. Crude HR represents relative hazard ratio. ${ }^{*}$. Adjusted HR represents multivariate-adjusted hazard ratio: age, sex, drinking, total bilirubin, albumin, white blood cell count, platelets, creatinine, blood urea nitrogen, international normalized ratio, prothrombin activity, alpha-fetoprotein, and HBV DNA. FZHY: Fuzheng Huayu capsule.

FZHY for the prevention of HCC development in patients with $\mathrm{HBC}$ are warranted. Second, patients may sometimes miss taking FZHY during the follow-up period. However, the FZHY group took medicine for at least 6 months (maximum, 60 months; median, 20 months). The influence on the study is minimal and may not have weakened 


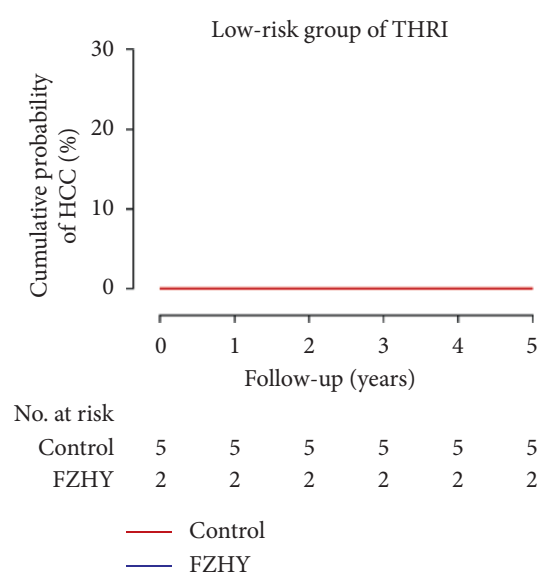

(a)
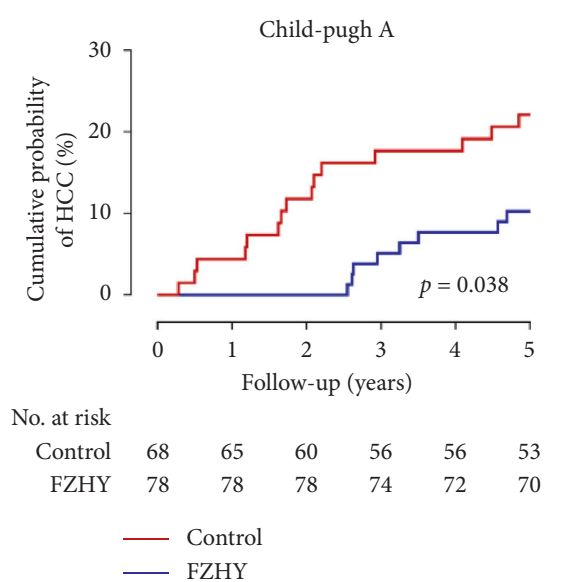

(d)

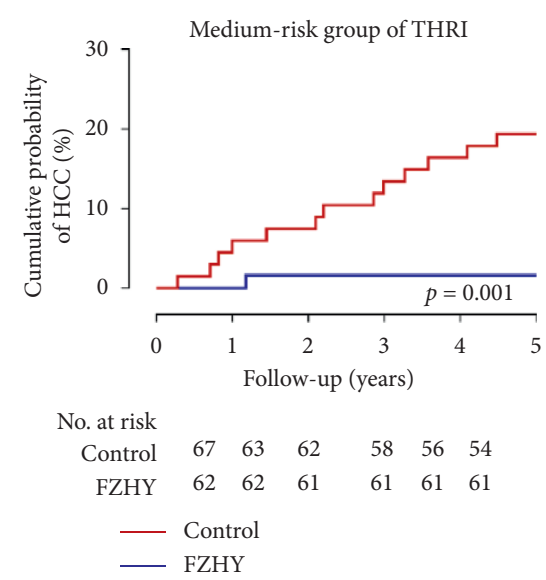

(b)

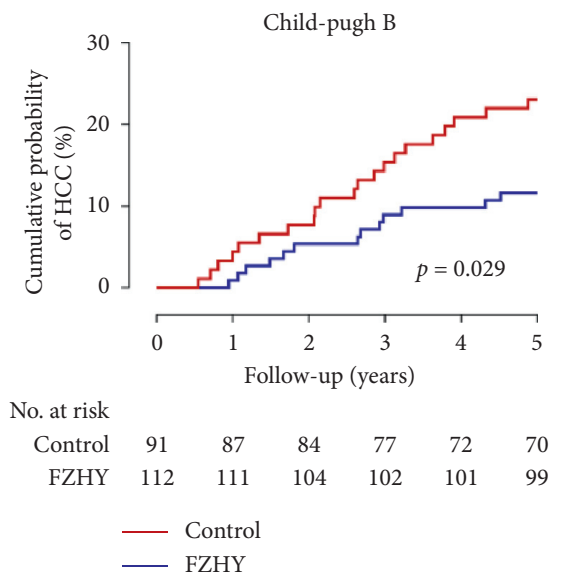

(e)

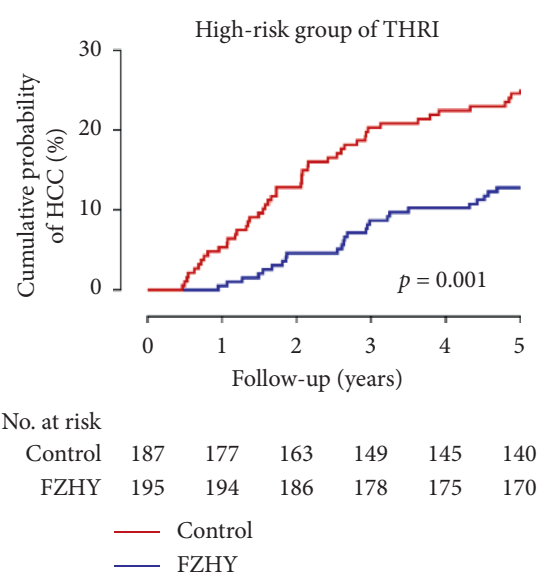

(c)

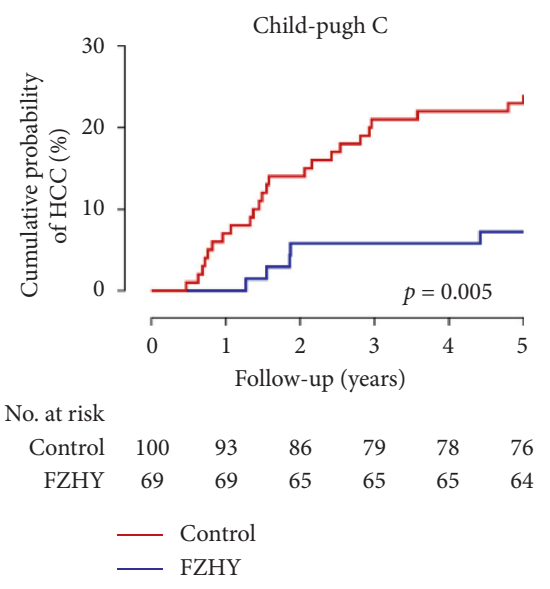

(f)

FIgURE 3: Subgroup analyses of the risk of HCC according to the THRI score and Child-Pugh classification. (a) Low-risk patients; (b) Medium-risk patients; (c) High-risk patients; (d) Child-Pugh A patients; (e) Child-Pugh B patients; (f) Child-Pugh C patients. HCC: hepatocellular carcinoma; THRI: Toronto HCC risk index.

the effect of FZHY. Third, alcohol consumption is an important factor in the development of HCC. In our study, it was not possible to accurately define the amount of alcohol consumed or to determine whether the patients abstained. It is necessary to reduce chronic exposure to hepatotoxic agents, such as alcohol, to maximize the cancer-preventive effects of antifibrosis TCM. Finally, although only a few $(24.1 \%)$ patients had previously received antiviral therapy, multivariate Cox proportional risk models were used to test the robustness of the results. FZHY group naïve to NAs were at a significantly lower risk of HCC (aHR 0.26, 95\% CI 0.10-0.66; $P=0.001$ ), as were FZHY users who had previously received NA treatment (aHR 0.43, 95\% CI 0.26-0.69; $P=0.005$ ).

\section{Conclusion}

The results of this 5-year follow-up cohort study suggested that FZHY plays an important role as an adjunct therapy to mitigate the development of HCC in patients with HBC. Further prospective cohort studies from multiple centers, however, are needed to verify this conclusion.

\section{Abbreviations}

FZHY: Fuzheng Huayu capsule

TCM: Traditional Chinese medicine

HCC: Hepatocellular carcinoma

HBC: Hepatitis B-caused cirrhosis

NAs: $\quad$ Nucleos (t)ide analogues

PS: $\quad$ Propensity score

CTP: Child-Turcotte-Pugh

THRI: Toronto HCC risk index

aHR: Adjusted hazard ratio

ETV: Entecavir

TDF: Tenofovir

CI: Confidence interval.

\section{Data Availability}

The data used to support the findings of this study are available from the corresponding author upon request. 


\section{Conflicts of Interest}

The authors declare that they have no conflicts of interest.

\section{Authors' Contributions}

KS and YL contributed to the project development and manuscript writing; XJW was responsible for data collection and manuscript modification; YXL, QZ, and YH collected and analyzed data; CPR, YYH, and JH collected data; XBW was responsible for project supervision and writing preparation and finalization. Ke Shi, Yao Liu, Xiaojing Wang contributed equally to this work.

\section{Acknowledgments}

This study was supported by the Capital Health Development Research Project (grant nos.2018-1-2172) and the Beijing Municipal Administration of Hospitals Clinical Medicine Development of Special Funding Support (No. ZYLX201707).

\section{Supplementary Materials}

Supplemental Table 1: components of the Toronto HCC Risk Index. (Supplementary Materials)

\section{References}

[1] G. Fattovich, F. Bortolotti, and F. Donato, "Natural history of chronic hepatitis B: special emphasis on disease progression and prognostic factors," Journal of Hepatology, vol. 48, no. 2, pp. 335-352, 2008.

[2] M. Omata, A. L. Cheng, N. Kokudo et al., "Asia-Pacific clinical practice guidelines on the management of hepatocellular carcinoma: a 2017 update," Hepatology International, vol. 11, no. 4, pp. 317-370, 2017.

[3] L. Xie, J. Yin, R. Xia, and G. Zhuang, "Cost-effectiveness of antiviral treatment after resection in hepatitis B virus-related hepatocellular carcinoma patients with compensated cirrhosis," Hepatology, vol. 68, no. 4, pp. 1476-1486, 2018.

[4] A. G. Singal and H. B. El-Serag, "Hepatocellular carcinoma from epidemiology to prevention: translating knowledge into practice," Clinical Gastroenterology and Hepatology, vol. 13, no. 12, pp. 2140-2151, 2015.

[5] C.-Y. Peng, R.-N. Chien, Y.-F. Liaw et al., "Hepatitis B virusrelated decompensated liver cirrhosis: benefits of antiviral therapy," Journal of Hepatology, vol. 57, no. 2, pp. 442-450, 2012.

[6] N. A. Terrault, N. H. Bzowej, K.-M. Chang, J. P. Hwang, M. M. Jonas, and M. H. Murad, "AASLD guidelines for treatment of chronic hepatitis B," Hepatology, vol. 63, no. 1, pp. 261-283, 2016.

[7] S. Köklü, Y. Tuna, M. T. Gulsen et al., "Long-term efficacy and safety of lamivudine, entecavir, and tenofovir for treatment of hepatitis B virus-related cirrhosis," Clinical Gastroenterology and Hepatology, vol. 11, no. 1, pp. 88-94, 2013.

[8] H. Kobashi, Y. Miyake, F. Ikeda et al., "Long-term outcome and hepatocellular carcinoma development in chronic hepatitis B or cirrhosis patients after nucleoside analog treatment with entecavir or lamivudine," Hepatology Research, vol. 41, no. 5, pp. 405-416, 2011.
[9] P. Arends, M. J. Sonneveld, R. Zoutendijk et al., "Entecavir treatment does not eliminate the risk of hepatocellular carcinoma in chronic hepatitis B: limited role for risk scores in Caucasians," Gut, vol. 64, no. 8, pp. 1289-1295, 2015.

[10] G. V. Papatheodoridis, H. L.-Y. Chan, B. E. Hansen, H. L. A. Janssen, and P. Lampertico, "Risk of hepatocellular carcinoma in chronic hepatitis B: assessment and modification with current antiviral therapy," Journal of Hepatology, vol. 62, no. 4, pp. 956-967, 2015.

[11] L. Zhang, G. Wang, W. Hou, P. Li, A. Dulin, and H. L. Bonkovsky, "Contemporary clinical research of traditional Chinese medicines for chronic hepatitis B in China: an analytical review," Hepatology, vol. 51, no. 2, pp. 690-698, 2010.

[12] K. S. Jung, S. U. Kim, K. Song et al., "Validation of hepatitis B virus-related hepatocellular carcinoma prediction models in the era of antiviral therapy," Hepatology, vol. 62, no. 6, pp. 800-806, 2015.

[13] Q. Cheng, N. Li, M. Chen et al., "Fuzheng Huayu inhibits carbon tetrachloride-induced liver fibrosis in mice through activating hepatic NK cellsfibrosis in mice through activating hepatic NK cells," Journal of Ethnopharmacology, vol. 145, no. 1, pp. 175-181, 2013.

[14] Y.-h. Jia, R.-Q. Wang, H.-M. Mi et al., "Fuzheng Huayu recipe prevents nutritional fibrosing steatohepatitis in micefibrosing steatohepatitis in mice," Lipids in Health and Disease, vol. 11, no. 1, p. 45, 2012.

[15] Y. N. Song, J. J. Sun, Y. Y. Lu et al., "Therapeutic efficacy of Fuzheng-Huayu tablet based traditional Chinese medicine syndrome differentiation on hepatitis-B-caused cirrhosis: a multicenter double-blind randomized controlled trail," Evidence-Based Complementary and Alternative Medicine, vol. 2013, Article ID 709305, 8 pages, 2013.

[16] J. Chen, Y. Hu, L. Chen, W. Liu, Y. Mu, and P. Liu, “The effect and mechanisms of Fuzheng Huayu formula against chronic liver disease," Biomedicine \& Pharmacotherapy, vol. 114, Article ID 108846, 2019.

[17] T. Y. Tsai, H. Livneh, T. H. Hung et al., "Associations between prescribed Chinese herbal medicine and risk of hepatocellular carcinoma in patients with chronic hepatitis B: a nationwide population-based cohort study," BMJ. Open., vol. 7, no. 1, Article ID e014571, 2017.

[18] S. Wu, Y. Kong, H. Piao et al., "On-treatment changes of liver stiffness at week 26 could predict 2-year clinical outcomes in HBV-related compensated cirrhosis," Liver International, vol. 38, no. 6, pp. 1045-1054, 2018.

[19] S. A. Sharma, M. Kowgier, B. E. Hansen et al., "Toronto HCC risk index: a validated scoring system to predict 10-year risk of HCC in patients with cirrhosis," Journal of Hepatology, vol. S0168-8278, no. 17, pp. 32248-32251, 2017.

[20] E. L. Ellis and D. A. Mann, "Clinical evidence for the regression of liver fibrosisfibrosis," Journal of Hepatology, vol. 56, no. 5, pp. 1171-1180, 2012.

[21] G. V. Papatheodoridis, S. Manolakopoulos, G. Touloumi et al., "Hepatocellular carcinoma risk in HBeAg-negative chronic hepatitis B patients with or without cirrhosis treated with entecavir: HepNet.Greece cohort," Journal of Viral Hepatitis, vol. 22, no. 2, pp. 120-127, 2015.

[22] T. Wang, X. Zhou, H. Liu et al., "FuzhengHuayu capsule as an adjuvant treatment for HBV-related cirrhosis: a systematic review and meta-analysis," Phytotherapy Research, vol. 32, no. 5, pp. 757-768, 2018.

[23] H. F. Huseini, S. M. Alavian, R. Heshmat et al., "The efficacy of Liv-52 on liver cirrhotic patients: a randomized, double-blind, 
placebo-controlled first approach," Phytomedicine, vol. 12, no. 9, pp. 619-624, 2005.

[24] S. Affo, L.-X. Yu, and R. F. Schwabe, "The role of cancerassociated fibroblasts and fibrosisin liver cancer," Annual Review of Pathology: Mechanisms of Disease, vol. 12, no. 1, pp. 153-186, 2017.

[25] G. Fattovich, T. Stroffolini, I. Zagni, and F. Donato, "Hepatocellular carcinoma in cirrhosis: incidence and risk factors," Gastroenterology, vol. 127, pp. S35-S50, 2004.

[26] B. Suh, S. Park, D. W. Shin et al., "High liver fibrosis index FIB-4 is highly predictive of hepatocellular carcinoma in chronic hepatitis B carriers," Hepatology, vol. 61, no. 4, pp. 1261-1268, 2015.

[27] T. Akima, M. Tamano, and H. Hiraishi, "Liver stiffness measured by transient elastography is a predictor of hepatocellular carcinoma development in viral hepatitis," Hepatology Research, vol. 41, no. 10, pp. 965-970, 2011.

[28] Q. L. Chen, Y. Y. Lu, J. H. Peng et al., "Dynamical regulation analysis Identifies molecular mechanisms of Fuzheng-Huayu formula against hepatitis B-caused liver cirrhosis," EvidenceBased Complementary and Alternative Medicine, vol. 2015, Article ID 238495, 9 pages, 2015.

[29] Y. Y. Tao, X. C. Yan, T. Zhou et al., "FuzhengHuayu recipealleviates hepatic fibrosis via inhibiting TNFlpha induced hepatocyte apoptosis," BMC Complementary and Alternative Medicine, vol. 14, no. 1, p. 449, 2014.

[30] H. Li, "Advances in anti hepatic fibrotic therapy with traditional Chinese medicine herbal formula," Journal of Ethnopharmacol. vol. 251, Article ID 112442, 2019. 